EPJ Web of Conferences 61, 07006 (2013)

DOI: 10.1051/epjconf/ 20136107006

(C) Owned by the authors, published by EDP Sciences, 2013

\title{
Faraday rotation measures in 20 AGN jets at parsec scale
}

\author{
Evgeniya V. Kravchenko ${ }^{1,2, a}$, Yuri Y. Kovalev ${ }^{1,3}$ and Kirill V. Sokolovsky ${ }^{1,4}$ \\ ${ }^{1}$ Astro Space Center of Lebedev Physical Institute, Profsoyuznaya 84/32, 117997 Moscow, Russia \\ ${ }^{2}$ Pushchino Radio Astronomy Observatory ASC Lebedev, Pushchino, 142290 Moscow region, Russia \\ ${ }^{3}$ Max-Planck-Institut für Radioastronomie, Auf dem Hügel 69, D-53121 Bonn, Germany \\ ${ }^{4}$ Sternberg Astronomical Institute, Moscow State University, Universitetsky pr., 13, 119991 Moscow, Russia
}

\begin{abstract}
We present multi wavelength parsec-scale Faraday rotation measure properties of twenty active galactic nuclei, observed with the Very Long Baseline Array simultaneously at 1.4, 1.6, 2.2, 2.4, 4.6, 5.0, 8.1, 8.4 and $15.4 \mathrm{GHz}$ in the full polarization mode. For the observed sources we construct Faraday rotation measure and Faraday-corrected linear polarization maps. Direction of electrical field in the optically thick core regions confirms bimodal distribution. No significant changes of a Faraday rotation measure transverse to the jet direction are found in any of the observed sources. We propose a new magnetic field spatial geometry reconstruction method based on core shift measurements. This technique is applied to the quasar $1004+141$. Results indicate an existence of a large scale poloidal magnetic field in the jet of 1004+141.
\end{abstract}

\section{Introduction}

Predicted [1] and observed [2] high linearly polarized emission in the jets of active galactic nuclei (AGN) provides evidence of a presence of highly ordered magnetic fields. Broad analytical and numerical simulations, performed during the last decade, deliver a detailed picture of AGN jets [3]. Considering general relativistic magneto hydro dynamics, these models predict helical shape of magnetic field: toroidally dominated in the vicinity of a black hole and poloidally dominated downstream. Shocks in a jet, jet interaction with the surrounding media or relativistic effects can enhance one of the field components, distorting the initial field direction, or tangle the field lines.

Intervening magnetized plasma, surrounding the jet, rotates the plane of an electromagnetic wave and can decrease the degree of polarization, distorting the inner jet characteristics from the ones observed from the Earth. The possible location of this plasma is external to the jet - boundary layer or sheath surrounding the jet, e.g., [4-6]. Sometimes internal Faraday rotation is needed to explain observations, e.g., [6]. Multi frequency Very Long Baseline Interferometry polarimetric observations can probe these regions, making possible to reconstruct inner jet characteristics and study Faraday media. In the case of external rotation, Faraday media rotates intrinsic polarization angle (EVPA) $\chi_{0}$ relative to the observed one $\chi$ linearly with the squared wavelength:

$$
\chi=\chi_{0}+R M \cdot \lambda^{2} \text {. }
$$

Here RM is the rotation measure $R M \sim \int n_{e} B d l\left(\mathrm{rad} / \mathrm{m}^{2}\right)$, which is proportional to the magnetic field component (parallel to the line of sight) $B$ and particle volume density $n_{e}$ along the line of sight $d l$.

We use a sample of 20 AGNs [7], observed with the VLBA quasi simultaneously at 9 frequencies in the range from 1.4 to $15.3 \mathrm{GHz}$ in the dual-polarization mode. All these sources (listed in Table 1) have bright jet components and large frequency dependent core shifts. Here and later we define the core at a given frequency as the optically thick apparent base of the jet. Application of the long baseline radio interferometer technique in our study is explained by not only the possibility to study sub-parsec scale regions of jets located in the vicinity of the central super-massive black hole, but also by sufficiently small depolarization of the signal within the telescope beam comparing to other radio telescopes.

Observations and data reduction are described in Section 2. In Section 3 we discuss our results of Faraday rotation measurements. Section 4 includes description of proposed method for a spatial magnetic field geometry reconstruction and results of its application. Section 5 summarizes our findings.

\section{Observations and data reduction}

\subsection{VLBA observations}

Sources were observed by the aperture synthesis array

\footnotetext{
ae-mail: evgenia.v.kravchenko@gmail.com
}

This is an Open Access article distributed under the terms of the Creative Commons Attribution License 2.0, which permits unrestricted use, distribution, and reproduction in any medium, provided the original work is properly cited. 
NRAO VLBA during four sessions in March-June 2007. The observing time for each target was about one hour per band. We have used used $\mathrm{L}, \mathrm{S}, \mathrm{C}, \mathrm{X}$ and $\mathrm{Ku}$ bands (according to the IEEE nomenclature), that corresponds to $21 \& 18,13,6,4$ and $2 \mathrm{~cm}$ respectively. Each band consists of four $8 \mathrm{MHz}$-wide frequency channels (IFs) per polarization. L, S, C and X bands were split into two subbands (see Table 2) and in the following analysis were processed independently.

Table 1. Source list.

\begin{tabular}{|c|c|c|}
\hline Source name & Redshift & $\begin{array}{c}\text { Observational } \\
\text { epoch }\end{array}$ \\
\hline $0148+274$ & 1.260 & 2007.03 .01 \\
\hline $0342+147$ & 1.556 & 2007.06 .01 \\
\hline $0425+048$ & 0.517 & 2007.04 .30 \\
\hline $0507+179$ & 0.416 & 2007.05 .03 \\
\hline $0610+260$ & 0.580 & 2007.03 .01 \\
\hline $0839+187$ & 1.272 & 2007.06 .01 \\
\hline $0952+179$ & 1.478 & 2007.04 .30 \\
\hline $1004+141$ & 2.707 & 2007.05 .03 \\
\hline $1011+250$ & 1.636 & 2007.03 .01 \\
\hline $1049+215$ & 1.300 & 2007.06 .01 \\
\hline $1219+285$ & 0.161 & 2007.04 .30 \\
\hline $1406-076$ & 1.493 & 2007.05 .03 \\
\hline $1458+718$ & 0.904 & 2007.03 .01 \\
\hline $1642+690$ & 0.751 & 2007.04 .30 \\
\hline $1655+077$ & 0.621 & 2007.06 .01 \\
\hline $1803+784$ & 0.680 & 2007.05 .03 \\
\hline $1830+285$ & 0.594 & 2007.03 .01 \\
\hline $1845+797$ & 0.056 & 2007.06 .01 \\
\hline $2201+315$ & 0.298 & 2007.04 .30 \\
\hline $2320+506$ & 1.279 & 2007.05 .03 \\
\hline
\end{tabular}

Observations were made in dual circular polarization mode with 2-bit sampling and total recording rate of 256 Mbps. The correlation of the data was performed with averaging time of $2 \mathrm{~s}$.

\subsection{Data calibration}

Data calibration and imaging, as well as model fitting were done in a standard manner for each frequency subband independently and are described in [7] in details. The amplitude calibration accuracy in the $1.4-15 \mathrm{GHz}$ frequency range is estimated to be $\sim 5 \%$ [7]. Polarization leakage-term calibration and absolute EVPA calibration were done in AIPS following the standard procedure, e.g., [8]. Final accuracy of EVPA was estimated to be $5^{0}$ at low frequencies (L-,S-bands), 2.5 $5^{0}$ at middle frequencies (C-,X-bands) and $2^{0}$ at the Ku-band.

\subsection{FRM map reconstruction method}

Because of substantial depolarization across the beam and changing opacity in some regions, dependence of EVPA vs. $\lambda^{2}$ was considered at low (1.4-2.4 GHz) and high (4.6-15.4 GHz) frequency intervals separately for all sources. Matching of the images' resolution was done by restoring the images with the same beam size, corresponding to the beam at the lowest frequency. Positions of optically thin jet components were used to aligned images at different frequencies.

Table 2. Observing bands.

\begin{tabular}{|c|c|c|}
\hline IEEE band & Sub-band 1 (MHz) & Sub-band 2 (MHz) \\
\hline $\mathrm{L}$ & $1404.5-1412.5$ & $1658.5-1666.5$ \\
\hline $\mathrm{S}$ & $2275.5-2283.5$ & $2383.5-2391.5$ \\
\hline $\mathrm{C}$ & $4604.5-4612.5$ & $4999.5-5007.5$ \\
\hline $\mathrm{X}$ & $8104.5-8112.5$ & $8425.5-8433.5$ \\
\hline $\mathrm{Ku}$ & \multicolumn{2}{|c|}{$15353.5-15377.5$} \\
\hline
\end{tabular}

We used an assumption that Faraday screen is external to the source, which means linear dependence of EVPA on square wavelength at different frequency intervals. We use only pixels with polarized flux density stronger than three times the polarization error (see [6] for a thorough discussion and references therein). Resolution of the $\mathrm{n} \pi$ wrap problem was done by minimizing reduced $\chi^{2}$. Finally, we used the $95 \%$ confidence limit for respective degrees of freedom. All these steps were realized in a perl script, created by the first author.

Observed Faraday rotations mainly happen in the media of our Galaxy and in the plasma surrounding extragalactic jets. Whereas the second one varies considerably at scales of source size, the first one is almost uniform at these scales. Thus, foreground contribution of the Galaxy was subtracted using the averaged Galactic RM map [9].

\section{FRM results}

\subsection{Linear polarization}

Two of the sources $(0610+260$ and $1845+797)$ do not show strong polarized flux density in the whole frequency range and were not considered in the analysis. Three AGNs are fully depolarized in the core region in the 1.4-15.4 GHz range, whereas they show strong linear polarization downstream the jet. In the 8.1-15.4 GHz interval the rest 15 targets show strong polarized flux density in the core region. In the 4.6-5.0 GHz range there are 13 sources with strongly polarized core and two AGNs with no significant core polarization. The observed 
low core linear polarization cannot be accounted for by an RM gradient across the beam [8]. In the 1.4-2.4 GHz interval only 10 out of 15 targets show significant linear polarization in the core. The rest 5 sources are depolarized and this can be explained by a depolarization in the beam.

\subsection{Faraday rotation measures}

We have succeeded in determining Faraday RM for all 18 sources. The assumption that Faraday rotation happens in the screen external to the source is confirmed for the majority of our targets with Faraday rotations of more than 45 degrees. In the $1.4-2.4 \mathrm{GHz}$ range $\mathrm{RM}$ values could not be evaluated in the core region of almost half of the observed sources, because of a superposition of several components, which we clearly see in the higher frequency maps. In one third of the sources no Faraday $\mathrm{RM}$ estimates could be obtained in the core regions. We attribute this to the smearing of a true EVPA in the beam because of a complex source structure in this region. All AGN jets show RM values in optically thin regions which do not change across the full observed frequency range. This is a good independent proof that we observe the same physical region at all wavelengths.

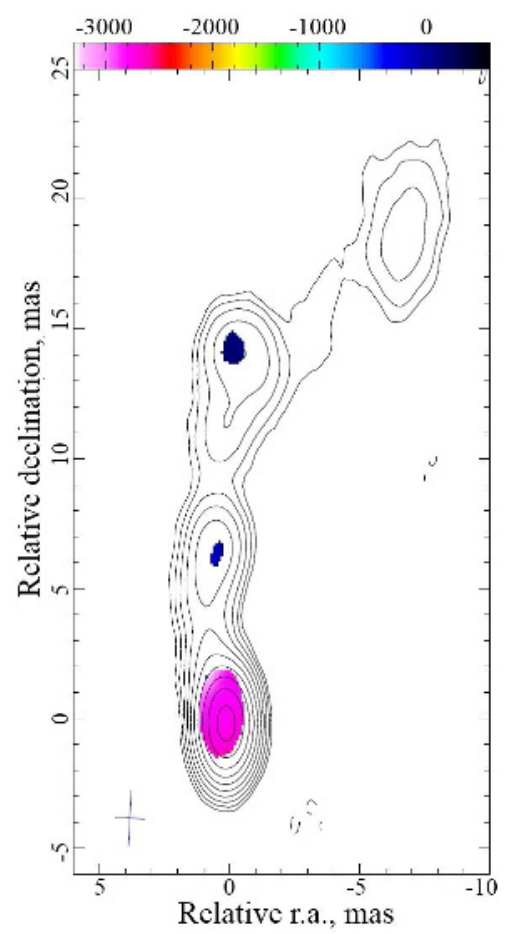

Figure 1. 8.1-15.4 GHz rotation measure map and $15.4 \mathrm{GHz}$ total intensity contours of $0952+179$, observed on 30 April 2007. The colour scale is in $\mathrm{rad} / \mathrm{m}^{2}$.

Using the relationship between the observed rotation measure $\mathrm{RM}_{\mathrm{obs}}$ and the rest frame value $\mathrm{RM}_{0}$ :

$$
R M_{o b s}=\frac{R M_{0}}{(1+z)^{2}},
$$

we estimate median values of $\mathrm{RM}_{0}: 75$ and $252 \mathrm{rad} / \mathrm{m}^{2}$ at low frequencies $(1.4-4.6 \mathrm{GHz})$ and 120 and $2000 \mathrm{rad} / \mathrm{m}^{2}$ at high frequencies $(4.6-15.3 \mathrm{GHz})$ for distant jet components and cores respectively. Thus we confirm the expected result, e.g., [10], that high RM values can be detected only at short wave lengths and in the cores, indicating the presence of stronger magnetic fields and denser medium upstream the jet. The highest measured $\mathrm{RM}$ in the source rest frame is $16911 \pm 76 \mathrm{rad} / \mathrm{m}^{2}$ in the $0952+179$ core as shown on the Figure 1.

Comparison of our RM measurements for several targets with results of other authors confirms previous findings $[6,8,11]$ : we do not see significant changes in the values of Faraday RM both in the core and distant components on time scales of several years. This supports the assumption that observed Faraday rotation occurs mainly in external to the jet medium. We note however, that we were able to measure RM only for cases of linear EVPA dependence on $\lambda^{2}$.

\subsection{Transversal FRM gradients}

If the helical magnetic field is present in the jet, its toroidal component will produce transverse Faraday RM gradient. So far this effect was found only in a handful of source and still remains controversial, e.g., $[6,12]$. The following two criteria were used during the analysis to avoid confusion between the real and spurious RM gradients $[3,6,12,13]$. We consider only transversal jet slices with the size $\geq 1.5$ times beam size and only regions located more than 1 beam size downstream the jet. Thus, we have analyzed transversal slices for 17 out of 36 RM maps. For seven of them we see changes in $\mathrm{RM}$ values at $1 \sigma$ level and for none of them by at least three times the typical error.

\section{Reconstruction of magnetic field geometry}

\subsection{Magnetic field direction in the core regions}

For 16 AGNs we were able to measure Faraday RM in the core region. After correcting for Faraday rotation, 11 targets show parallel E-field to the jet direction in the core region, and 5 sources - perpendicular. Such bimodal behavior has already been observed, e.g., [12] and references therein. It can be produced by illuminating front and rear of emitting regions of jets with different pitch angles in the presence of a helical magnetic field or by a longitudinal magnetic field and superposition of optically thick and thin regions within one beam width.

\subsection{D magnetic field geometry}

All our targets show flat or inverted spectra over the full frequency range in the core region which reflects opacity conditions in this region. In the case of synchrotron radiation of a self-absorbed region, the polarization angle is parallel to the magnetic field direction.

Assuming the magnetic field strength inversely depends on the distance from jet origin and squared 
decrease of electron density with a distance from the central engine [14], one can recalculate observed positions of optically thick core components at different frequencies to an absolute distance. Thus we can recover the 3D geometry of magnetic field in the jet base. As an example, reconstructed magnetic field geometry is shown on Figure 2 for $1004+141$ in the $1.4-8.4 \mathrm{GHz}$ range, sky plane projection. Assuming the $1004+141$ jet observing angle of 6 degrees, our results reveal an existence of a large-scale poloidal magnetic field in this source at least over 120 pc.

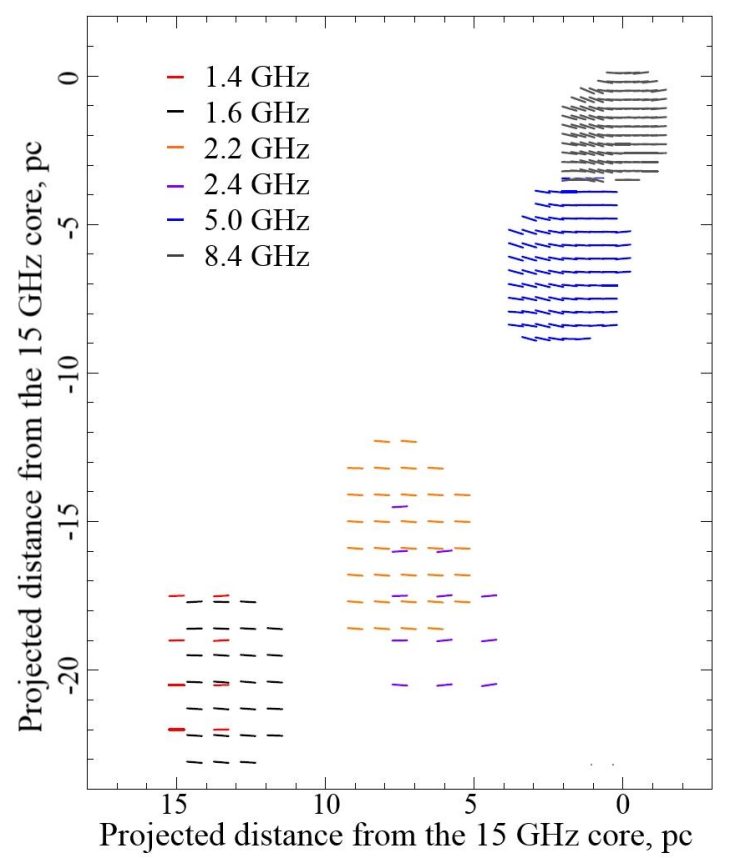

Figure 2. 1.4-8.4 GHz intrinsic EVPA orientation geometry in the core region of $1004+141$.

\section{Summary}

For the first time we have measured Faraday RMs for 20 AGN jets through 9 frequency bands in $1.4-15.3 \mathrm{GHz}$ range. Our results provide a unique opportunity to study $\mathrm{RM}$ in AGN parsec-scale core regions in a wide frequency range. Faraday rotation is found to be external to the source screen in the majority of our targets. All AGN jets show RM values in optically thin jet regions which do not change with frequency. We did not find significant Faraday RM gradient transverse to the jet direction in any of the targets. We have made first attempt to spatially reconstruct the intrinsic magnetic field geometry in the observed jet base. Results for $1004+141$ indicate presence of a poloidal magnetic field.

\section{Acknowledgments}

This research was supported by Russian Foundation for Basic Research, project № 12-02-33101 and by Research Program OFN-17 of the Division of Physics, Russian Academy of Sciences. The VLBA is an instrument of the
National Radio Astronomy Observatory, a facility of the National Science Foundation operated under cooperative agreement by Associated Universities, Inc. This research has made use of data from the University of Michigan Radio Astronomy Observatory (for EVPA calibration) which has been supported by the University of Michigan and by a series of grants from the National Science Foundation, most recently AST-0607523

\section{References}

[1] Burn, B.J., MNRAS, 133, 67 (1966)

[2] Lister, M. L. \& Homan, D. C., AJ, 130, 1389 (2005)

[3] Broderick, A. E. \& McKinney, J. C., ApJ, 725, 750 (2010)

[4] Zavala, R. T. \& Taylor, G. B., ApJ, 612, 749 (2004)

[5] Attridge, J. M., et al, ApJ, 633, 85 (2005)

[6] Hovatta, T., et al, AJ, 144, 105 (2012)

[7] Sokolovsky, K. V. Et al, A\&A, 532, 38 (2011)

[8] Zavala, R. T. \& Taylor, G. B., ApJ, 589, 126 (2003)

[9] Taylor, A. R., et al, ApJ, 702, 1230 (2010)

[10] Trippe, S., et al, A\&A, 540, 74 (2012)

[11] O'Sullivan, S. P. \& Gabuzda, D. C., MNRAS, 400, 26 (2009)

[12] Algaba, J. C., MNRAS, 429, 3551 (2013)

[13] Taylor, G. B. \& Zavala, R. T., ApJ, 722, 183 (2010)

[14] Lobanov, A. P., A\&A, 330, 79 (1998) 